\title{
The eye - a window to cardiovascular diseases
}

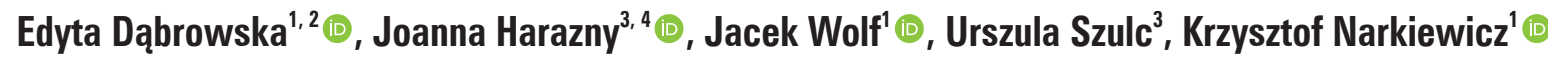 \\ 'Department of Hypertension and Diabetology, Medical University of Gdansk, Gdansk, Poland \\ ${ }^{2}$ First Department of Cardiology, Medical University of Gdansk, Gdansk, Poland \\ ${ }^{3}$ Department of Human Physiology and Pathophysiology, University of Warmia and Mazury in Olsztyn, Olsztyn, Poland \\ ${ }^{4}$ Clinical Research Centre, Department of Nephrology and Hypertension, Friedrich-Alexander University Erlangen-Nürnberg, Erlangen, Germany
}

\begin{abstract}
Microvascular alterations can parallel and even precede development of cardiovascular disease. Ocular bulb, due to transparent anatomical elements provides a unique opportunity to examine microcirculation non-invasively and in vivo by means of scanning laser Doppler flowmetry. This device enables to obtain both structural and functional parameters of retinal microvasculature. Alterations observed in retinal arterioles, i.e. lumen narrowing, increased wall-to-lumen ratio, rarefaction or reduced retinal capillary flow, have been independently associated with cardiometabolic diseases: hypertension, heart failure, coronary artery disease, stroke, chronic kidney disease and diabetes. Therefore, examination of easily accessible retinal microcirculation may provide novel insights into the mechanisms underlying various diseases, serve as a marker of target organ damage and add valuable information to cardiovascular risk stratification.
\end{abstract}

Key words: microcirculation; retinal arterioles; remodeling

Arterial Hypertens. 2020, vol. 24, no. 2, pages: 56-60

DOI: $10.5603 /$ AH.a2020.0006

\section{Introduction}

Cardiovascular diseases remain the most important cause of mortality worldwide [1] despite an impressive development of novel treatment strategies. Substantial proportion of cardiovascular events can only partially be explained by typical risk factors. Therefore, we should constantly focus on studying new biomarkers that would enable more accurate risk stratification and earlier therapeutic interventions [2].

Circulatory system consists of three inseparable components: micro-, macrocirculation and the heart. These three interrelated districts influence structure and function of each other, regulating hemodynamics of circulatory system $[3,4]$.

\section{Coupling between}

microcirculation-macrocirculation-the heart

To understand the coupling between vascular tree and the heart, we propose the following diagram (partially based on diagram created by Laurent $S$. and Agabiti-Rosei E. [5]), demonstrating consecutive cardiovascular changes occurring in hypertension:

In small arteries, alterations in essential hypertension correspond to increased wall thickness, decreased lumen diameter and increased wall-to-lumen ratio without any change in total wall material and

Address for correspondence: Edyta Dąbrowska

Department of Hypertension and Diabetology, Medical University of Gdansk, Smoluchowskiego 17, Gdansk 80-214, Poland,

tel: (+48) 5858444 40, fax: (+48) 5858444 50; e-mail: edabrowska@gumed.edu.pl 


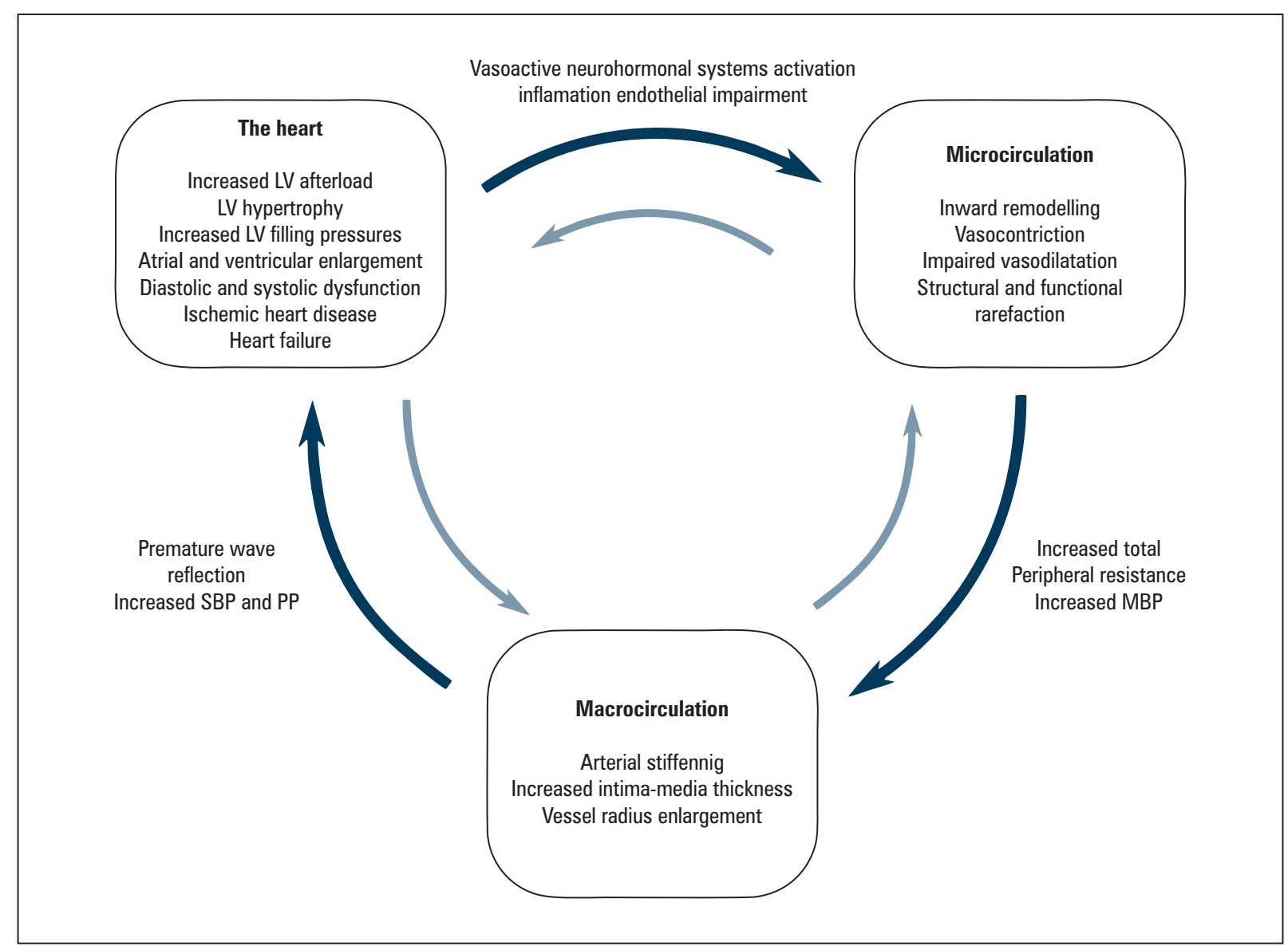

Figure 1. Coupling between microcirculation, macrocirculation and the heart. LV — left ventricular; MBP — mean blood pressure; $\mathrm{PP}$ — pulse pressure; RAAS — renin-angiotensin-aldosterone system; SBP — systolic blood pressure

were called by Mulvany et al. in 1996 as eutrophic inward remodeling [6]. These changes are major determinants raising total peripheral resistance and mean blood pressure. In consequence, stiff components load in vessel wall, exaggerating arterial stiffening and premature wave reflection which, arriving in late systole, augments central systolic and pulse pressures [7]. Importantly, as aortic stiffness increases, stiffness gradient decreases leading to the shift of reflection sites toward microvessels [8]. This contributes to the penetration of excessive pressure pulsatility into microcirculation and then to small artery remodeling and damage [9].

Moreover, increased central systolic BP contributes to greater left ventricular afterload, and consequent myocardial hypertrophy and increased myocardial oxygen demand [10]. Elevated values of $\mathrm{PP}$ and decreased DBP are related to diminished coronary arteries perfusion during diastole thereby leading to myocardial ischemia. Additionally, chronically elevated blood pressures result in microvascular remodeling and vasoconstriction that may also impair vasodilatory reserve and lead to myocardial hypoperfusion [10].

Myocardial hypertrophy accompanied by intensive fibrosis augments LV filling pressures that precede atrial and ventricular enlargement and further diastolic and systolic dysfunction. Subsequent increased activity of vasoactive neurohormonal systems such as sympathetic nervous system or reninangiotensin-aldosterone system - on the one hand preserve circulatory homeostasis, on the other hand promote inflammation processes, endothelial dysfunction and further vascular remodeling $[11,12]$.

\section{The microvasculature of the eye}

Previous studies have shown that changes in microcirculation can parallel $[13,14]$ and even precede cardiovascular diseases [15]. Therefore, the microvascular area appears to be a valuable tool to assess cardiovascular risk especially at asymptomatic stage of disease. 


\section{Microcirculation - anatomy}

Microcirculation is a part of the circulatory system localized between the arterial and venous system. It includes vessels with a diameter less than $150 \mu \mathrm{m}$ : arterioles, metaarterioles, capillaries, venules, lymphatic vessels and arteriovenous anastomoses. The peripheral circulation of the whole body consists of 10 billion capillaries with a total surface approximately 500-700 square meters. [16].

\section{Microcirculation - role in total peripheral resistance}

Poiseuille's law states that vascular resistance $(\mathrm{R})$ is directly proportional to the length $(\mathrm{L})$ of the vessel and the viscosity $(\eta)$ of the blood, and inversely proportional to the fourth power of the vessel radius to $\left(\mathrm{r}^{4}\right)$ :

$$
R=(8 L \eta) / \pi r^{2}
$$

According to this equation and taking into account the huge number of microvessels in the whole body and microvascular inward remodeling described by Mulvany et al., we may easily infer that microcirculation is the pivotal modulator of total peripheral resistance. It is worth noting that different types of remodeling, i.e. hypertrophic, eutrophic or hypotrophic, are observed in various diseases. Additionally, remodeling can be inward or outward.

\section{Microcirculation of retina - measurement of structure and function}

From all microvascular beds, retinal microcirculation due to transparent anatomical elements of ocular bulb, provides a unique opportunity to be examined non-invasively and in vivo. Scanning laser Doppler flowmetry allows evaluating both structure and function of microcirculation. The examination is performed in a dark room, in a sitting position, after 15 min of rest, without any pharmacological pupil dilatation. Measurements are taken in juxtapapillary area of the right eye, $2-3 \mathrm{~mm}$ temporally superior to the optic nerve. The retinal sample of $2.56 \times 0.64$ $\times 0.3 \mathrm{~mm}$ at a resolution of 256 points $\times 64$ lines $\times 128$ lines is scanned within 2 seconds [17].

Retina is illuminated by laser light (of 670 $\mathrm{nm}$ wavelength), which scattered by moving red blood cells is shifted in frequency by an amount $\Delta f$ - shown in equation 1 :

$$
\Delta f:(1 / 2 \pi)\left(k_{s c}-k_{i}\right) v
$$

where $k_{s c}$ is the wave vector of the scattered light, $k_{i}$ is the wave vector of the incident light and $v$ is the velocity vector of the moving particle. The laser beam hits moving blood cells and the spectrum broadens. The collected light is guided to a photodetector and then analyzed.

The effective Doppler shift, called flow is calculated in equation 2:

$$
\omega=\frac{2 \pi}{P(f=0)}\left(\sum_{125}^{2,000 \mathrm{~Hz}} f P(f) d f\right)
$$

where $f P(f) d f$ is the power of the photodetector associated with fluctuations in a frequency range $d f$ about $f$ and $P(f=0)$ is the power in the detected signal. Flow describes total distance travelled by the moving red blood cells per unit time within the sample volume [18].

Perfusion map is obtained from images by means of Automatic Full Field Analysis of Perfusion (AFFPIA) [17].

The following structural parameters of retinal arterioles are evaluated: outer diameter (OD) is assessed in reflection images, and the lumen diameter (LD) is assessed in perfusion images [17].

Wall thickness (WT) is calculated using the formula:

$$
W T=O D-L D / 2
$$

Wall cross-sectional area (WCSA) is calculated using the formula:

$$
W C S A=(\pi / 4) \times(O D 2-L D 2)
$$

WLR is calculated using the formula:

$$
W L R=(O D-L D) / L D
$$

\section{Microvascular retinal alterations in cardiovascular diseases}

\section{Hypertension}

In the study of Smith et al., generalized retinal arteriolar narrowing was significantly associated with subsequent 5-year incident severe hypertension, independent of cardiovascular risk factors and baseline blood pressure status [19]. Also increased value of retinal arteriolar wall-to-lumen ratio was widely revealed as a hallmark of hypertension [20]. Apart from structural microvascular changes, retinal capillary blood flow (RCF) was found to be significantly reduced in hypertension. Ritt et al. have reported that in response to flicker light, the increase of RCF was significantly lower in hypertensive patients than 
in normotensive subjects, identifying a reduced vasodilatatory properties and risk of tissue hypoperfusion in hypertension [21]. Moreover, it was recently shown that RCF significantly decreases with hypertension advancement expressed by elevated value of pulse wave velocity [22].

\section{Heart diseases}

Wong et al. have reported in a population-based cohort study including 11612 participants that the presence of retinopathy was associated with significantly higher incidence of congestive heart failure $(15.1 \%$ in subjects with retinopathy vs $4.8 \%$ in subjects without retinopathy $(\mathrm{p}<.001)$. After adjustment for typical cardiovascular risk factors, the presence of retinopathy was associated with even 2-fold higher risk of congestive heart failure in 7-year observation [15]. The recent investigations have also reported that lumen narrowing of retinal vessels is independently associated with left atrium enlargement and left ventricular mass - the antecedents of diastolic dysfunction and heart failure [13].

In the study of Wang et al., the narrower retinal arterioles were related to lower hyperemic myocardial blood flow and lower perfusion reserve evaluated by cardiac magnetic resonance in asymptomatic patients with no coronary calcification. These results revealed that retinal changes may serve as a marker of coronary microvascular disease [23].

\section{Stroke}

The main finding of the study of Harazny et al. was the evidence of retinal hypertrophic remodeling in hypertensive patients with cerebrovascular event. In this group, microvascular changes were expressed by significant decrease in lumen diameter, increase in wall cross sectional area and wall-to-lumen ratio compared to normotensive subjects [14]. In the investigation of Lindley et al., patients with lacunar stroke were more likely to have changes in retinal microvasculature, such as focal arteriolar narrowing, arteriovenous nipping, enhanced light reflex of the arteriolar wall, generalized retinal arteriolar narrowing, small arteriole:venule ratio and retinal venular widening [24].

\section{Microvascular retinal alterations in other diseases}

\section{Chronic kidney disease}

Patients with chronic kidney disease have greater wall thickness and greater wall-to-lumen ratio of retinal arterioles in comparison to healthy indi- viduals. Moreover, intercapillary distance reflecting capillary rarefaction was significantly greater in the chronic kidney disease group compared to the healthy control group. After adjustment for age, gender, BMI and serum cholesterol, wall thickness, wall cross sectional area, wall-to-lumen ratio and intercapillary distance remained significantly higher in chronic kidney disease group [25]. Furthermore, Ritt et al. have demonstrated that retinal remodeling was related to microalbuminuria - the marker of impaired renal function and well established risk factor for cardiovascular mortality and morbidity. The results of the study have revealed that wallto-lumen ratio of retinal arterioles was positively related to urinary albumin-to-creatinine ratio [26]. Moreover, the investigation of Sabanayagam et al., carried out in a large multiethnic population, has shown a close relationship between the presence of retinal arteriolar narrowing and the increased likelihood of chronic kidney disease defined by an estimated glomerular filtration rate less than $60 \mathrm{~mL} / \mathrm{min}$ per $1.73 \mathrm{~m}^{2}$ [27].

\section{Diabetes}

In the prospective study of adult Australians, narrower retinal arterioles were associated with increased incidence of diabetes, independently of fasting plasma glucose, systolic blood pressure, family history of diabetes, waist circumference, and other confounding factors. These findings indicate that early microvascular alterations may underlie the development of diabetes and serve as a subclinical marker of diabetes risk [28]. Moreover, in the study of Jumar et al., diabetes was linked to a greater retinal arteriolar wall thickness and wall cross sectional area than in hypertensive or healthy subjects indicating for hypertrophic remodeling [29].

\section{Conclusions}

Retinal circulation provides a window to other microvascular beds. Its complex evaluation appears to be a valuable tool to better understand mechanisms underlying cardiometabolic diseases and to assess development and progression of vascular damage. Identification of asymptomatic subjects with altered retinal microcirculation might provide an opportunity for early therapeutic intervention. Thus, extending the list of typical diagnostic tools with the new microvascular parameters could allow more precise assessment of subclinical target organ damage, and improve prevention and treatment of cardiovascular diseases. 


\section{Funding}

The study was supported by the Ministry of Science and Higher Education - Young Investigators Funding 01-0267/08/125.

\section{References}

1. Mc Namara K, Alzubaidi H, Jackson JK. Cardiovascular disease as a leading cause of death: how are pharmacists getting involved? Integr Pharm Res Pract. 2019; 8: 1-11, doi: 10.2147/IPRP.S133088, indexed in Pubmed: 30788283.

2. Dzau VJ. Markers of malign across the cardiovascular continuum: interpretation and application. Circulation. 2004; 109(25 Suppl 1): IV1-IV2, doi: 10.1161/01.CIR.0000133445.78855.aa, indexed in Pubmed: 15226244.

3. Struijker Boudier H. From Macrocirculation to Microcirculation: Benefits of Preterax. Am J Hypertens. 2007; 20(7): S15-S18, doi: 10.1016/j.amjhyper.2007.04.013.

4. Lee ML, Rosner BA, Weiss ST. Relationship of blood pressure to cardiovascular death: the effects of pulse pressure in the elderly. Ann Epidemiol. 1999; 9(2): 101-107, doi: 10.1016/s10472797(98)00034-9, indexed in Pubmed: 10037553.

5. Nilsson PM, Olsen MH, Laurent S. Early Vascular Aging (EVA): New Directions in Cardiovascular Protection. Academic Press 2015.

6. Mulvany MJ, Baumbach GL, Aalkjaer C, et al. Vascular remodeling. Hypertension. 1996; 28: 505-506, indexed in Pubmed: 8794840.

7. O'Rourke M. Arterial stiffness, systolic blood pressure, and logical treatment of arterial hypertension. Hypertension. 1990; 15(4): 339347, doi: 10.1161/01.hyp.15.4.339, indexed in Pubmed: 2180816.

8. Briet M, Boutouyrie P, Laurent $S$, et al. Arterial stiffness and pulse pressure in CKD and ESRD. Kidney Int. 2012; 82(4): 388-400, doi: 10.1038/ki.2012.131, indexed in Pubmed: 22534962.

9. Mitchell GF. Effects of central arterial aging on the structure and function of the peripheral vasculature: implications for end-organ damage. J Appl Physiol (1985). 2008; 105(5): 1652-1660, doi: 10.1152/ japplphysiol.90549.2008, indexed in Pubmed: 18772322.

10. Laurent $S$, Boutouyrie P. The structural factor of hypertension: large and small artery alterations. Circ Res. 2015; 116(6): 10071021, doi: 10.1161/CIRCRESAHA.116.303596, indexed in Pubmed: 25767286.

11. Chatterjee K. Neurohormonal activation in congestive heart failure and the role of vasopressin. Am J Cardiol. 2005; 95(9A): 8B-813B, doi: 10.1016/j.amjcard.2005.03.003, indexed in Pubmed: 15847852 .

12. Harvey A, Montezano AC, Touyz RM. Vascular biology of ageingImplications in hypertension. J Mol Cell Cardiol. 2015; 83: 112-121, doi: 10.1016/j.yjmcc.2015.04.011, indexed in Pubmed: 25896391.

13. Dąbrowska E, Harazny J, Miszkowska-Nagórna E, et al. Lumen narrowing and increased wall to lumen ratio of retinal microcirculation are valuable biomarkers of hypertension-mediated cardiac damage. Blood Press. 2019; 29(2): 70-79, doi: 10.1080/08037051.2019. 1657769, indexed in Pubmed: 32228237.

14. Harazny JM, Ritt M, Baleanu D, et al. Increased wall:lumen ratio of retinal arterioles in male patients with a history of a cerebrovascular event. Hypertension. 2007; 50(4): 623-629, doi: 10.1161/HYPERTENSIONAHA.107.090779, indexed in Pubmed: 17698722.

15. Wong TY, Rosamond W, Chang PP, et al. Retinopathy and risk of congestive heart failure. JAMA. 2005; 293(1): 63-69, doi: 10.1001/ jama.293.1.63, indexed in Pubmed: 15632337.
16. Guyton AC, Hall JE. Textbook of medical physiology. Elsevier Inc, Philadelphia 2006.

17. Harazny JM, Raff U, Welzenbach J, et al. New software analyses increase the reliability of measurements of retinal arterioles morphology by scanning laser Doppler flowmetry in humans. J Hypertens. 2011; 29(4): 777-782, doi: 10.1097/HJH.0b013e328343c27a, indexed in Pubmed: 21297501.

18. Michelson G, Schmauss B, Langhans MJ, et al. Principle, validity, and reliability of scanning laser Doppler flowmetry. J Glaucoma. 1996; 5(2): 99-105, indexed in Pubmed: 8795741.

19. Smith W, Wang JJ, Wong TY, et al. Retinal arteriolar narrowing is associated with 5-year incident severe hypertension: the Blue Mountains Eye Study. Hypertension. 2004; 44(4): 442-447, doi: 10.1161/01.HYP.0000140772.40322.ec, indexed in Pubmed: 15302843 .

20. Gallo A, Mattina A, Rosenbaum D, et al. Retinal arteriolar remodeling evaluated with adaptive optics camera: Relationship with blood pressure levels. Ann Cardiol Angeiol (Paris). 2016; 65(3): 203-207, doi: 10.1016/j.ancard.2016.04.021, indexed in Pubmed: 27184511.

21. Ritt M, Harazny JM, Ott C, et al. Impaired increase of retinal capillary blood flow to flicker light exposure in arterial hypertension. Hypertension. 2012; 60(3): 871-876, doi: 10.1161/HYPERTENSIONAHA.112.192666, indexed in Pubmed: 22777934.

22. Dąbrowska E, Harazny JM, Miszkowska-Nagórna E, et al. Aortic stiffness is not only associated with structural but also functional parameters of retinal microcirculation. Microvasc Res. 2020; 129: 103974, doi: 10.1016/j.mvr.2020.103974, indexed in Pubmed: 31923388.

23. Wang Lu, Wong TY, Sharrett AR, et al. Relationship between retinal arteriolar narrowing and myocardial perfusion: multi-ethnic study of atherosclerosis. Hypertension. 2008; 51(1): 119-126, doi: 10.1161/HYPERTENSIONAHA.107.098343, indexed in Pubmed: 17998474.

24. Lindley RI, Wang JJ, Wong MC, et al. Multi-Centre Retina and Stroke Study (MCRS) Collaborative Group. Retinal microvasculature in acute lacunar stroke: a cross-sectional study. Lancet Neurol. 2009; 8(7): 628-634, doi: 10.1016/S1474-4422(09)70131-0, indexed in Pubmed: 19481977.

25. Bosch A, Scheppach JB, Harazny JM, et al. Retinal capillary and arteriolar changes in patients with chronic kidney disease. Microvasc Res. 2018; 118: 121-127, doi: 10.1016/j.mvr.2018.03.008, indexed in Pubmed: 29559377.

26. Ritt M, Harazny JM, Ott C, et al. Wall-to-lumen ratio of retinal arterioles is related with urinary albumin excretion and altered vascular reactivity to infusion of the nitric oxide synthase inhibitor N-monomethyl-L-arginine. J Hypertens. 2009; 27(11): 2201-2208, doi: 10.1097/HJH.0b013e32833013fd, indexed in Pubmed: 19625969.

27. Sabanayagam C, Shankar A, Koh D, et al. Retinal microvascular caliber and chronic kidney disease in an Asian population. Am J Epidemiol. 2009; 169(5): 625-632, doi: 10.1093/aje/kwn367, indexed in Pubmed: 19092170.

28. Nguyen TT, Wang JJ, Islam FM, et al. Retinal arteriolar narrowing predicts incidence of diabetes: the Australian Diabetes, Obesity and Lifestyle (AusDiab) Study. Diabetes. 2008; 57(3): 536-539, doi: 10.2337/db07-1376, indexed in Pubmed: 18086902.

29. Jumar A, Ott C, Kistner I, et al. Early Signs of End-Organ Damage in Retinal Arterioles in Patients with Type 2 Diabetes Compared to Hypertensive Patients. Microcirculation. 2016; 23(6): 447-455, doi: 10.1111/micc.12291, indexed in Pubmed: 27270643. 